\title{
Non-pharmacological management for delirium
}

\begin{abstract}
Delirium is an acute attention and cognitive disorder, frequently presented in the elderly by multifactorial causes, which has a serious impact on mortality, health costs and quality of life.1 Non-pharmacological measures are the standard of prevention and treatment of delirium. This approaches such as HELP (Hospital Elder Life Program) include cognitive stimulation techniques, however, we have observed in our Hospital, that the "Day Room", based on Snoezelen sensory stimulation theory, improves delirium severity. This article describes a clinical case of a female geariatric patient with delirium, whom receives both programs (HELP and Snoezelen), where we observed a decrease on delirium severity scales. For the above, we can conclude that "Day Room" might be a very useful non-pharmacological instrument on patients with delirium, so we should do comparative research that sustains clinical efficacy.
\end{abstract}

Volume 3 Issue 2 - 2018

\author{
Natalia Capistrán Páramo \\ Hospital General de México, México
}

Correspondence: Natalia Capistrán Páramo, Hospital General de México, México, Tel +(044)5534880764,

Email nat.capistran@gmail.com

Received: February 21, 2018 | Published: March 07, 2018

Keywords: delirium, acute confusional state, non-pharmacological treatment, "Day Room", neurocognitive stimulation, sensory stimulation

Abbreviations: HELP, hospital elder life program; MDAS, memorial delirium assessment scale; DRS-R-98, delirium rating scale revised

\section{Introduction}

Delirium, an acute decline in attention and cognition, is a common life-threatening, and potentially preventable clinical syndrome among persons who are 65 years of age or older. ${ }^{1}$ Prevalence is highest among those who are frail or patients who are critically unwell or at the end of life. Over the age of 80 years, more than one third of those in hospital will experience delirium. ${ }^{2}$ The cause of delirium is typically multifactorial. In fact, the development of delirium involves the complex interrelationship between a vulnerable patient (with a predisposing factor) and exposure to precipitating factors or noxious insults. ${ }^{3}$ some of the main predisposing factors are: age of 65 or older, dementia, cognitive impairment and functional dependence, visual and hearing impairment and coexisting medical conditions. And precipitating factors such as: Drugs, primary neurologic diseases, infections, severe acute illness, dehydratation, surgery, use of physical restraints, use of catheters. ${ }^{1-3}$

\section{Pathophysiology}

Rather, accumulating evidence suggests that several different sets of interacting biological factor result in disruption of largescale neuronal networks in the brain, leading to acute cognitive dysfunction. $^{4}$ Some of the leading hypothesized mechanisms contributing to delirium includes neurotransmitters, inflammation, physiologic stressors, metabolic derangements, electrolyte disorders, and genetic factors. ${ }^{5}$ The list of potential neurotransmitters involved in delirium is long, but a relative cholinergic deficiency and dopamine excess are the most commonly inferred. ${ }^{6}$

\section{Clinical features and diagnosis}

The hallmark of delirium is an acute impairment of cognition with a fluctuating course. It includes change in the level of consciousness, ranging from a hyperactive state with prominent agitation, hypervigilance and combativeness to a hypoactive state characterized by lethargy, stupor or even coma. Multiple cognitive domains can be affected, with attention being invariably involved, and orientation, memory, language, visuospatial and executive function are also often impaired. $^{7}$

The current reference standard diagnostic criteria are the 5 th edition of American Psychiatric Association's Diagnostic and Statistical Manual of Mental Disorders (DSM-5) and WHO's International Classification of Diseases, 10th Revision (ICD-10), but the most widely used instrument for identification of delirium is the Confusion Assessment Method (CAM), which has been validated in high-quality studies with sensitivity of $94 \% .{ }^{5}$ Although there are other validated instruments such as Global Attentiveness Rating (GAR), Clinical Assessment of Confusion (CAC), Delirium Observation Screening Scale (DOSS), Memorial Delirium Assessment Scale (MDAS), Delirium Rating Scale Revised-98 (DRS-R-98). ${ }^{8}$

\section{Treatment}

Primary prevention with non-pharmacological multicomponent approaches is widely accepted as the most effective strategy for delirium. ${ }^{9-11}$ The Hospital Elder Life Program (HELP), a multicomponent intervention strategy with proven effectiveness and cost-effectiveness in the prevention of delirium and functional decline through targeting of risk factors for delirium is the most widely disseminated approach. The interventions include reorientation, therapeutic activities, reduced use and doses of psychoactive drugs, early mobilization, and promotion of sleep, maintenance of adequate hydration and nutrition, and provision of vision and hearing adaptations. The intervention prevented the initial development of delirium and reduced the total number of days of delirium. Once an initial episode of delirium had occurred, however, the intervention had no significant effect on the severity of delirium or on the likelihood of recurrence. ${ }^{12}$ HELP is now implemented in more than 200 hospitals worldwide, but adaptations and alternatives may be necessary in some settings.

Other non-pharmacological strategies include proactive old age medicine consultation, multifactorial targeted interventions, staff training, interventions delivered by family members and mobility or rehabilitation interventions, the use of earplugs at night, ${ }^{13}$ non- 
pharmacological sleep protocols. ${ }^{14}$ Delirium rooms,${ }^{15}$ and other spaces that provide restraint-free care for patients with delirium, are staffed with specially trained nurses, and promote non-pharmacological management approaches, are an intriguing idea for provision of specialized management for patients with delirium, but have not yet been assessed in a controlled trial. ${ }^{5}$

The general purpose of this case presentation is to open the discussion, to the probability of implementing improves to the nonpharmacological delirium treatment. Our patient receives cognitive and sensory stimulation (based on Snoezelen stimulation) in "day room", doing brain stimulation activities, so this way it is planned to identify a positive response on delirium severity.

\section{Case presentation}

The patient was born in Mexico City, attended 2 years of primary school, currently unemployed, widowed five years ago, never had any children, cared for by her nephew, she is dependent for basic activities (Katz 1/6 preserves feeding, Lawton brody 0/8). She uses a walker after having suffered a cerebrovascular accident (CVA).

Her medical records indicated hypertension treated with enalapril $10 \mathrm{mg} \mathrm{q} 12 \mathrm{hr}$ and metoprolol $50 \mathrm{mg} \mathrm{q} 12 \mathrm{hr}$; diabetes mellitus type 2, in treatment with metformin $850 \mathrm{mg} \mathrm{q12} \mathrm{hr}$, paroxystic atrial fibrillation anticoagulated with acenocoumarin $1 \mathrm{mg}$ q24 hr, right middle cerebral artery ischemic stroke on 2013 and 2015, causing as sequels, leftsided hemiparesis and epilepsy in treatment with levetiracetam 1g q12 hr.

Requires a knee prothesis two years ago due to osteoarthritis. She fell a month ago from her own height, causing a right fronto-temporal contusion, after it, she presents clonic movements of left hemibody, with no alteration of wakefulness, managed with midazolam 10 $\mathrm{mg} I V$, single dose with crisis referral, brain tomography is done and shows encephalomalacia in frontal and temporal lobe and frontoparietal chronic subdural hematoma, with no surgery criteria, deciding treatment with carbamazepine and discharged after 72 hours. Two days later, she presents hyporexia, vomit in one occasion and periods of disorientation alternated with periods of lucidity and visual hallucinations, incoherent speech and psychomotor agitation, next day she presents tonic movements of two minutes long with no loss of wakefulness, for what she is taken to ER in which she's found with blood pressure of 180/120 mmHg, $120 \mathrm{bpm}$, dehydrated, arrhythmic, with disorientation and psychomotor agitation, handle begins with beta blocker, IECA and antagonist calcium, for every agitation period $5 \mathrm{mg}$ of haloperidol and hospitalization is decided in charge of geriatrics where urinary tract infection is detected, treated with amikacin, severity of delirium is measured with MDAS obtaining 21/30. Next day she presents $160 / 90 \mathrm{mmHg}$ blood pressure. FC 96 bpm Gluc 156, she receives sensory stimulation based on Snoezelen model in "day Room" for about 3 hours where she paints, cooperating $60 \%$ of time, severity of delirium is measured with MDAS obtaining $15 / 30$. The day after she presents $140 / 80$ blood pressure, FC $88 \mathrm{bpm}$, goes to "Day Room" for sensory and cognitive stimulation, where she plays "lotería" (sort of Bingo), word games, orientation and categories for 4 hours, cooperating $80 \%$ of time, severity of delirium is measured with MDAS obtaining 10/30, reducing from severe to mild delirium in 72 hours.

\section{Discussion}

As mentioned before, angular treatment of delirium is based on non-pharmacological measures in which many recommendations, with no scientific evidence or clinical trials had been done. However, HELP program was developed which refers that is only useful as prevention, with no significance on severity or recurrence once the first episode is presented.

The HELP program, based on the study of multicomponent intervention, determined the presence of delirium, according to CAM criteria, severity of delirium was measured by adding a numeric value for every of the four designed symptoms: fluctuation, disorganized thinking and consciousness alteration. Assigned punctuation was: missing 0 points, mild 1 point or strong 2 points, except fluctuation which was determined as: missing 0 points or present 1 point. The sum of these values allows to obtain scores from $0-7$, the higher the score, the greater the severity. ${ }^{5}$

Nonetheless, other mind-altered functions were not evaluated, so the use of scales such as MDAS or DRS-R98 is recommended, since they evaluate other affected functions such as consciousness, orientation, short term memory impairment, digit span, attention, disorganized thinking, perceptual disturbance, delusions, psychomotor activity and sleep-wake cycle disturbance (for MDAS 1996).

In this case we base the treatment, according to scientific evidence of Snoezelen sensory stimulation, which report wheter a combined treatment comprised of standard psychiatric inpatient care and a non-pharmacological intervention, multi-sensory behavior therapy, reduces agitation and apathy and improves ADLs in people with dementia on an acute care psychiatric hospital unit compared to standard psychiatric inpatient care alone. ${ }^{16}$ This, based on the operant paradigm of automatic reinforcement and the physiological model of the relaxation respone. ${ }^{17}$ Even though this sensory stimulation has implemented only in patients with dementia, we believe is a good alternative for adding to the multicomponent management in patients with acute delirium.

This patient receives sensorial stimulation in two times, with 3 and 4 hours per session, finding an important improvement in severity of delirium based on MDAS scale.

We need to validate this theory in randomized control trials to obtain statistical significance, nevertheless, it could open an alternative for management of patients with delirium, an so, validate the effectiveness in complications such as hospital stay, cognitive impairment and functionality, but also factors that interfere with quality of life, that are not usually measured like collapse of the caregiver, adherence to treatment, hospital cooperation, a recurrence decrease in pharmacological or restrain handle, recalling that the patient medical treatment shall also include psychological and social measures.

\section{Acknowledgements}

None.

\section{Conflict of interest}

The authors declare no conflict of interest.

\section{References}

1. Sharon SK. Delirium in Older Persons. $N$ Engl $\mathrm{J}$ Med. 2006;354(11);1157-65.

2. Todd OM1, Teale EA. Delirium: a guide for the general physician. Clinical Medicine. 2017;17(1):48-53.

3. Rolfson D. The causes of delirium. In: Lindesay J, Rockwood K, Macdonald A, editors. Delirium in old age. Oxford, England: Oxford University Press; 2002:101-22.

4. Watt D, Koziol K, Budding D. Delirium and confusional states. In: 
Noggle C, Dean R, editors. Disorders in Neuropsychiatry. New York: Springer Publishing Company; 2012.

5. Inouye SK, Westendorp RG, Saczynski JS. Delirium in elderly people. Lancet. 2014;383(9920):911-922.

6. Hshieh TT, Fong TG, Marcantonio ER, et al. Cholinergic Deficiency Hypotesis in Delirium: A Synthesis of Current Evidence. J Gerontol A Biol Sci Med Sci. 2012;63(7):764-772.

7. Vanja CD, Andrew JS. Delirium. Continuum Lifelong Learning Neurol. 2010;16(2):120-134.

8. Camilla LW, Jayna HL, David LS. Does This Patient Have Delirium?Value of Bedside Instruments. JAMA. 2010;304(7):779786.

9. Mahony OR, Murthy L, Akunne A. Synopsis of the national institute for health and clinical excellence guideline for prevention of delirium. Ann Intern Med. 2011;154(11):746-51.

10. Inouye SK. Delirium in older persons. NEngl J Med. 2006;354:115765.

11. Wei LA, Fearing MA, Sternberg EJ, et al. The confusion assessment method: a systematic review of current usage. J Am Geriatr Soc. 2008;56(5):823-830.

12. Inouye SK, Bogardus ST, Charpentier PA, et al. A multicomponent intervention to prevent delirium in older patients. $N$ Engl J Med.
1999;340(9):669-76.

13. Van RB, Elseviers MM, Van DW, et al. The effect of earplugs during the night on the onset of delirium and sleep perception: a randomized controlled trial in intensive care patients. Crit Care. 2012;16(3):R73.

14. Mc DJA, Mion LC, Lydon TJ, et al. A nonpharmacologic sleep protocol for hospitalized older patients. J Am Geriatr Soc. 1998; 46(6):700-05.

15. Flaherty JH, Steele DK, Chibnall JT, et al. An ACE unit with a delirium room may improve function and equalize length of stay among older delirious medical inpatients. J Gerontol A Biol Sci Med Sci. 2010;65(12):1387-92.

16. Staal JA, Sacks A, Matheis R, et al. The effects of Snoezelen (multisensory behavior therapy) and psychiatric care on agitation, apathy, and activities of daily living in dementia patients on a short term geriatric psychiatric inpatient unit. Int'l Psychiatry In medicine. 2007;37(4):357-370.

17. Benson H, Klipper M. The relaxation response. New York: Avon Books; 1975. 